\title{
Implementing a computer algebra system in Haskell
}

\author{
José Romildo Malaquias ${ }^{\text {a }}$, Carlos Roberto Lopes ${ }^{\mathrm{b}, *}$ \\ a Departamento de Computação, Universidade Federal de Ouro Preto, Ouro Preto, Brazil \\ ${ }^{\mathrm{b}}$ Faculdade de Computação, Universidade Federal de Uberlândia, Uberlândia, Brazil
}

\begin{abstract}
There are basically two kinds of mathematical computation, numerical and symbolic. Numerical algorithms are usually implemented in strongly typed languages, and compiled with a view to efficiency. Symbolic algorithms are mostly written for interpreters in untyped languages. Therefore, symbolic mathematics is usually slow, and bug ridden. Since symbolic algorithms are usually more difficult to implement, there are also very few computer algebra systems. This paper presents a computer algebra system that is both fast, and implemented in a strongly typed language, and designed to accept compiled extensions. The authors describe a scheme to achieve these goals without creating difficulties for the end-user. The reason for creating this new computer algebra system is to make feasible mixed computation, i.e., programming software that needs both numerical computation and computer algebra. For instance, Finite Element Methods require a lot of number crunching as well as computer algebra to perform triangularization, manipulating shape functions, etc. Mixed computation requires speed and safety that interpreted computer algebra cannot provide.
\end{abstract}

(c) 2007 Elsevier Inc. All rights reserved.

Keywords: Computer algebra; Mixed computation; Functional programming; Haskell

\section{Introduction}

Computers have been used for numerical computations since their beginning. These general purpose machines can also be used for transforming and combining symbolic expressions as well. That is, computers can be used not only to deal with numbers, but also with abstract symbols representing mathematical formulas. This fact was realized much later and is only now gaining acceptance among mathematicians and engineers.

According to Winkler [1] in his short introduction to Computer Algebra, before 1850 mathematicians solved the majority of their problems by extensive calculations. However in the 19th century, the style of mathematical research changed from quantitative to qualitative aspects. The advent of modern digital computers and the development of Computer Algebra programs in the 20th century stressed this trend. Notwithstanding even today many mathematicians think that the role of computers is number crunching and their role

\footnotetext{
* Corresponding author.

E-mail address: crlopes@ufu.br (C.R. Lopes).
} 
is the application of the appropriate algebraic transformations to the problem and thus underestimating the power of computing systems for algebraic manipulation, which was recognized as early as 1844 by Lady Augusta Ada Byron, countess of Lovelace. In describing the possible applications of the Analytical Engine developed by Charles Babbage, she wrote [1]:

"Many people who are not conversant with mathematical studies imagine that because the business of [Babbage's Analytical Engine] is to give its results in numerical notation, the nature of its process must consequently be arithmetical and numerical rather than algebraic and analytical. This is an error. The engine can arrange and combine its numerical quantities exactly as if they were letters or any other general symbols; and in fact it might bring out its results in algebraic notation were provisions made accordingly."

Indeed a modern computer is a universal machine that is able to carry out an arbitrary algorithm, being it numerical, symbolic or of any other nature.

What does one mean by Computer Algebra or symbolic algebra computation? R. Loos wrote [2]:

"Computer Algebra is that part of computer science which designs, analyzes, implements, and applies algebraic algorithms."

Algebraic algorithms are algorithms that utilize the rules of Algebra to transform algebraic formulas containing numbers, variables and function applications. In short, Computer Algebra systems deal not only with integers and reals, but also with variables representing unknown quantities and any expression combining numbers and variables by means of mathematical operations. Numbers are represented exactly. The approximations of floating point numbers for reals are not acceptable anymore. So there is no need to be concerned about approximation errors.

Computer Algebra software finds application in any field dealing with the formulation of laws in mathematical terms, using algebraic equations and/or analytic concepts such as ordinary, partial differential equations and integrals. After the formulation of a law, it should be solved, simplified or reduced to the normal form. This process can be performed with help of Computer Algebra. Normal form is a concept that changes from one branch of Mathematics to another. For instance, in Lambda Calculus, a term in the normal form has no subterms of the form $(\lambda x . R S)$, i.e., terms that can be lambda reduced. In relational algebra, one may have another definition. The fact is that the existence of the normal form makes it possible to compare expressions. After a sequence of transformation, one expects that two equivalent expressions be reduced to the same normal form. In this work, the reader will find a definition of normal form that allows one to compare two semantically equivalent algebraic expressions.

Nowadays there are a handful of programs which perform Computer Algebra. These programs can be applied in areas such as Mathematics, Computer Science, Engineering, Economics, etc. With these programs one can perform algebraic simplifications as well as handle literals, as illustrated by the following examples.

$$
\begin{aligned}
& 5 \frac{a^{2}-2 a b+b^{2}}{a-b}=5 a+5 b, \\
& \frac{\mathrm{d}}{\mathrm{d} x}\left(a x^{2}+b x+c\right)=2 a x+b, \\
& \sin ^{2} a+\cos ^{2} a=1 .
\end{aligned}
$$

Most of these programs suffer drawbacks. For instance, their programming languages are not referentially transparent, that is, they do not allow substitution of equals for equals, invalidating the use of certain Mathematical laws. Even if other areas of thought are able to do without substitution of equals for equals, this is not true of Mathematics, the main subject of Computer Algebra. Therefore, the absence of referential transparency is a mortal sin, when it occurs in a Computer Algebra language.

The notion of transparency is due to Whitehead and Russell [3]. Antoni Diller [4] illustrates the invalidation of substitution in natural languages with the following sentence drawn from Russell [5]:

George IV wished to know whether Scott was the author of Waverley. 
It should be noted that the above sentence is true, since the mad king really wished to know whether Scott was the author of Waverley. However, we do know that Scott and the author of Waverley are indeed the same person. Therefore, we may feel entitled to substitute Scott for the author of Waverley in Russell's sentence. In doing so, we get

George IV wished to know whether Scott was Scott.

The result is obviously false, for even being mad, the King knew well that Scott was Scott.

Imperative computer languages suffer from the same lack of transparency as natural languages. For example, one cannot make use of the commutative property of multiplication to conclude that the $\mathrm{C}$ program of listing 1 will print the same number twice, although $f(2) \times g(5)=g(5) \times f(2)$ in Mathematics. This happens because $\mathrm{C}$ functions may change the state of the computing system (by means of assignments to variables) besides (possibly) computing a value. In listing 1 function $g$ changes the contents of the global variable $k$ before returning a value and function $f$ needs the value of $k$ in order to compute its result. So calls to $g$ have an effect on calls to $f$. The return value of $f$ depends on the previous calls to $g$. This program outputs $-6=99$ and shows that $C$ is a referentially opaque language. Thus it is highly desirable that the language used to express Computer Algebra algorithms be referentially transparent.

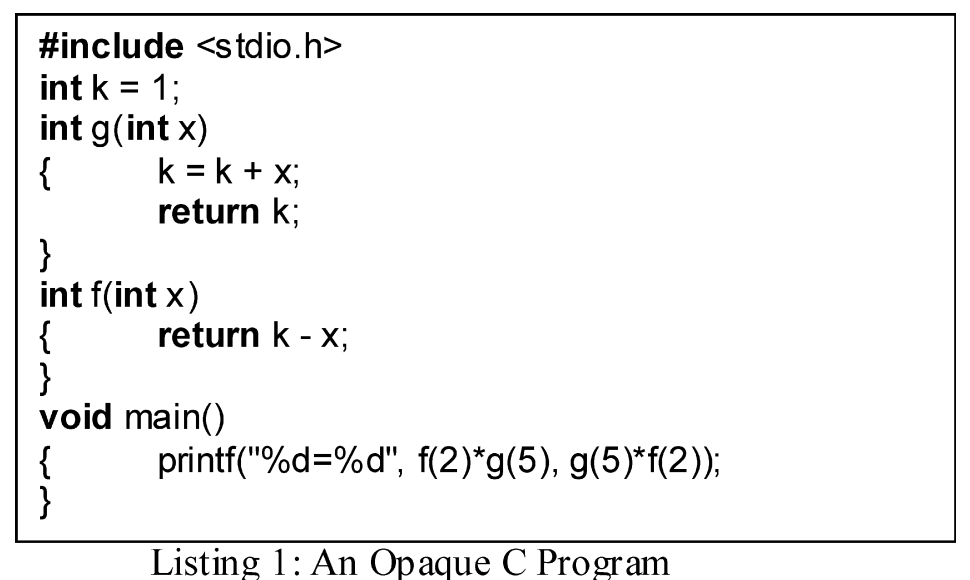

This work has three phases. The authors started by implementing a computer algebra system in Scheme, an imperative language of the LISP family. This was done so as to become familiar with computer algebra algorithms by performing a traditional implementation. Besides this, a working Scheme system could be used for comparison, and prototyping. The next step was to implement a Computer Algebra library for Haskell, a language with the desired referential transparency property, highly popular, and quite flexible, which facilitates the creation of novel algorithms. The Haskell implementation was used as a proof of concept. The next and last step was to translate the Haskell implementation to Clean, a highly efficient language of the same family as Haskell. Then the main purpose of this work is to provide algorithms for manipulation of algebraic expressions in a declarative context, compatible with Mathematics, and implement them as a language for Computer Algebra programming embedded in Clean. Its implementation is one more example that shows that functional languages are viable for day to day programming, even beating conventional languages in some aspects, such as the level of abstraction, and even in speed, when the algorithms involve memory manipulation and data structures.

One can ask the reason for translating the Computer Algebra system described in this paper from Haskell to Clean, since the two languages are close implementations of a branch of Mathematics called Lambda Calculus, and show referential transparency. Clean is much faster than Haskell, and this is enough to justify the translation, if one can show that speed is at a premium. Since people do a lot of algebraic manipulation by hand, it seems that speed is not very necessary in this branch of Mathematics. However, the fact is people limit themselves to relatively small problems. For instance, Delaunay completed a hand calculation in 1867 to determine the moon's position as a function of time; it took him 10 years to complete the task, and a further 
10 years to check it (vide [6]). One hundred years after Delaunay's efforts Henrard and Rom [7] used a rather primitive computer algebra program to check Delaunay's work. According to Henrard in a private communication to us, their Computer Algebra program was able to get the result in less than $24 \mathrm{~h}$. The program was almost 1000 times faster than Delaunay. The conclusion is that for purely algebraic problems the speed of available Computer Algebra systems is more than enough. However, numerical calculations often have a strong symbolic component. For instance, the Finite Element Method requires handling shape functions, performing triangularization, and finding a node renumbering schemes that produce narrow band matrices. Since these tasks may be performed concurrently with numerical calculations, an efficient Computer Algebra system may be useful.

The development of this library has also lead us to identify some limitations of Haskell and Clean, as well as extensions to these languages that would make the implementation easier and more elegant. These limitations are described in the paper through examples drawn from the library.

The rest of this paper is organized as follows. Section 2 presents related works in the area, describing the most popular Computer Algebra Systems. Section 3 provides a short overview of functional programming languages, which are derived from the Lambda Calculus. Section 4 describes some relevant aspects of the system design and implementation. Section 5 discusses some limitations in Haskell that make the library more difficult to design and implement. Finally, Section 6 concludes the discussion.

\section{Related work}

According to Nilsson [8], research in Computer Algebra had its beginnings when James Slagle created the program Saint, which was able to integrate functions by elementary methods. After this first step, Joel Moses improved the algorithms of Saint, creating another program of symbolic integration, which was named SIN.

In the sixties, Carl Engleman, Joel Moses and William Martin (at the Massachusetts Institute of Technology, as part of the MAC project) started the project Macsyma ${ }^{1}$ [9], which is one of the finest systems of Symbolic Mathematics. It is quite large, and written in Lisp. After Macsyma, many other systems of Computer Algebra came to light. Each one of these systems tried to correct a real or imaginary weakness of Maclisp, the original computer language of Macsyma. Some of these systems are:

- Reduce. ${ }^{2}$ In the beginning, Lisp had many dialects. In general, every machine had its own dialect. Macsyma was developed in Maclisp. Therefore, one could not build the system in a machine running Franz Lisp, Interlisp, etc. The designers of Reduce $[10,11]$ proposed a standard dialect of Lisp, which should be portable to any machine. Although Reduce became very popular, Standard Lisp was badly beaten by Common Lisp in the struggle to become the standard dialect of the main AI language. In the meantime, Macsyma migrated from MACLISP to Common Lisp. The result is that Macsyma runs in a standard language, while Reduce is built on top of an obscure dialect known as Standard Lisp.

- Maple. ${ }^{3}$ Lisp always had a bad reputation for being slow. Therefore, Maple [12] was developed (at Waterloo University, Canada) in a procedural language (C) with a view to greater efficiency. Since procedural languages like $\mathrm{C}$ are not fit for symbolic computation, the implementors of Maple were forced to design a symbolic language. Not being experts in compiler construction, they wrote an interpreter for the Maple language. The result is that Maple is much slower than Macsyma and other Lisp based systems. Maple's source code is not in the public domain.

- Derive. ${ }^{4}$ This package (and its precursor MuMATH) was designed to offer a small and fast Computer Algebra system [13]. It was the only competitor to Macsyma that really delivered on its promises. The system is indeed very small and reasonably fast. However, it did not meet the commercial success of Reduce and Maple.

\footnotetext{
${ }^{1}$ http://www.macsyma.com/

2 http://www.rrz.uni-koeln.de/REDUCE

3 http://www.maplesoft.com/

${ }^{4}$ http://www.derive.com/
} 
Maple, Reduce and Derive were designed to be worthy competitors of Macsyma. There are also systems designed to offer limited functionality in a friendly environment. Among these systems, MatLab ${ }^{5}[14]$ and Mathematica $^{6}[15]$ became very popular. It is interesting to note that these two systems that promised limited functionality also failed to deliver on their promise. Due to demands from the market, the implementors of both Matlab and Mathematica have increased the functionality of their systems, which soon became as fat as Reduce. However, neither Matlab nor Mathematica have the well designed architecture of Reduce. In fact, these systems suffered from a chaotic growth.

\section{A short overview of functional programming}

In this section we describe the main concepts related to functional programming. Functional programming has roots in the lambda calculus. We start by describing the syntax and semantics of the lambda calculus. After we show that lambda calculus presents all the features existent in modern functional programming languages such as Haskell and Clean. In reality a pure functional programming language is an applied lambda calculus.

\subsection{Lambda calculus}

Functional programming has roots in the lambda calculus. The lambda calculus is a branch of logic, developed in the 1920s and 1930s by logicians who wanted to explore how to define functions formally and how to use this formalism as a foundation for mathematics. The lambda calculus is a simple and powerful formal language of functions. The first developments were made by Schönfinkel [16], and Curry [17] who defined a variation called combinatory logic. Later, Church [18] defined the first version of the actual lambda calculus. Note that these early logicians had no intention of defining any programming languages. Actually, there weren't even any computers at that time. The syntax of the lambda calculus is very simple, because there are only three types of constructs: variables, abstractions, and applications. A brief notion of the meaning of each one of the constructs (intuitive semantics) follows:

1. The variables are just place holders for values. In this section we use lowercase letters for representing variables such as $x, y, f, g$.

2. Abstraction is a model for function definition, which is not associated to any name. In terms of syntax an expression $\lambda x . M$ denotes an abstraction. The variable $x$ is the parameter of the function, and the expression $M$ is the body of the function. All occurrences of $x$ in $\lambda x . M$ are said to be bound. All unbound occurrences of a variable in a term are free.

3. Applications are a model for the computation of a functional value. In a lambda expression like (M1 M2), the first item (called the rator, from operator) is applied to the second item (called the rand from operand).

In a lambda term like ( $M 1 M 2$ ), if $M 1$ (sometimes called the applicand) is an abstraction, the term may be reduced. $M 2$, the argument, may be substituted into the body of $M 1$ in place of the formal parameter of $M 1$ and the result is a new lambda term which is equivalent to the old one. As we mentioned earlier, if a lambda term contains no subterms of the form $\left(\begin{array}{ll}\lambda x . M 1 & M 2\end{array}\right)$ then it cannot be reduced, and is said to be in a normal form. The expression $\{N / x\} M$ represents the result of taking the term $M$ and replacing all free occurrences of $x$ with $N$. Thus we write

$$
(\lambda x . M \quad N) \rightarrow\{N / x\} M
$$

The right side $\{N / x\} M$ is considered to be simpler than $(\lambda x . M N)$. This reduction is known as $\beta$-reduction. There is an additional rule called $\alpha$-conversion, which renames bound variables:

$$
\lambda x . M \rightarrow \lambda y \cdot\{y / x\} M \quad y \text { not free in } M
$$

\footnotetext{
${ }^{5} \mathrm{http}: / /$ www.mathworks.com/

${ }^{6} \mathrm{http}: / /$ www.mathematica.com/
} 
A $\beta$-reduction reduction captures the essential behavior of all mathematical functions. For instance, consider the function that computes the square of a number. We might write

The square of $x$ is $x * x$

In the specification above we use the symbol ' $*$ ' to indicate multiplication. The symbol $x$ is the formal parameter of the function. To evaluate the square for a particular argument, say 2, we insert it into the definition in place of the formal parameter:

The square of 2 is $2 * 2$

In order to evaluate the resulting expression $2 * 2$ it is necessary to know what the number 2 means and how to multiply two numbers. Since any computation is simply a composition of the evaluation of suitable functions on suitable primitive arguments, this simple substitution principle suffices to capture the essential mechanism of computation.

With respect to the previous example it is important to notice that the number 2 and multiplication operator ' $*$ ' belong to arithmetic and are not part of the pure calculus. We are going to use the term constant in the next subsection to describe such constructs. However, in the lambda calculus, notions such as '2' and '*' can be represented without any need for externally defined primitive operators or constants. It is possible to identify terms in the lambda calculus, which, when suitably interpreted, behave like the number 2 and like the multiplication operator.

Computation in the lambda calculus is symbolic. A term is "reduced" into the simplest form as possible. A reduction strategy for the lambda calculus is a rule for choosing redexes ("reduction expressions"). A redex is a term of the form $\left(\begin{array}{ll}\lambda x . M & N\end{array}\right)$. There are two reductions strategies, which are known as call-by-value and callby-name.

The call-by-name reduction strategy chooses the leftmost-outermost redex while the call-by-value reduction strategy chooses the leftmost-innermost redex in a term. The expressions inner and outer refer to nesting of terms. The call-by-name strategy is also referred to as normal-order reduction. If there exists a normal form then the call by value reduction strategy is going to find it. On the other hand, call-by-value strategy can get stuck, forever evaluating an argument that will never be used. Next we show the reduction of the expression $(\lambda x \cdot x+x)(2 * 2)$ by using both strategies.

Call-by-name strategy:

$$
\begin{aligned}
(\lambda x \cdot x+x)(2 * 2) & \rightarrow(2 * 2)+(2 * 2) \\
& \rightarrow 4+(2 * 2) \\
& \rightarrow 4+4 \\
& \rightarrow 8
\end{aligned}
$$

Call-by-value strategy:

$$
\begin{aligned}
(\lambda x \cdot x+x)(2 * 2) & \rightarrow(\lambda x \cdot x+x) 4 \\
& \rightarrow 4+4 \\
& \rightarrow 8
\end{aligned}
$$

It is possible to combine call-by-name evaluation with updating. By using call-by-name an expression is evaluated only when its value is needed. Updating means that if the value of an expression is needed more than once, the result of the first evaluation is stored and subsequent requests for it will return the stored value immediately without further evaluation. The resulting strategy is known as lazy evaluation.

\subsection{Applied lambda calculus: functional programming languages}

The syntax of the applied lambda calculus or a functional programming language is also very simple. The syntax of the lambda calculus described above is extended by including a new construct: constants. Constants correspond to the built-in constants and operators in a programming language. Some examples of constants 
are: true, false, if. The intended use of a constant is formalized by defining reduction rules. Reduction rules for constants are called $\delta$ rules. The following lines describe two reduction rules.

$$
\begin{aligned}
& \text { if True } M N \rightarrow M \\
& \text { if false } M N \rightarrow N
\end{aligned}
$$

The symbol $\rightarrow$ should be read as "is reduced to". The following example makes use of the rules defined above. Consider a combinator or defined by $\lambda x y$.ifxtruey. A combinator is a lambda term without free variables. The or combinator resembles an important construction in programming languages: the if-then-else statement. The remaining rules simulate integer arithmetic.

$$
\begin{aligned}
& \text { if true } M N \rightarrow M \\
& \text { if false } M N \rightarrow N \\
& \text { iszero } 0 \rightarrow \text { true } \\
& \text { iszero }\left(\operatorname{succ}^{k} 0\right) \rightarrow \text { false where } k \geqslant 1 \\
& \text { iszero }\left(\text { pred }^{k} 0\right) \rightarrow \text { false where } k \geqslant 1 \\
& \text { succ }(\text { pred } M) \rightarrow M \\
& \text { pred }(\text { succ } M) \rightarrow M
\end{aligned}
$$

The constants succ and pred correspond to the successor and predecessor functions, respectively. The notation $M^{k} N$, for $k>+0$, corresponds to $k$ successive applications of $M$ to $N$. An important feature in applied lambda calculus is how to define a recursive function, which is a function defined in terms of itself. Recursion is achieved by means of fixed-point combinators. $M$ is a fixed-point combinator if $M f=f(M f)$. Next we are going to use the fixed-point combinator designated by $Y$ to introduce recursivity into the language. The definition of $Y$ follows:

$$
Y=\lambda f \cdot(\lambda g . f(g g))(\lambda g \cdot f(g g))
$$

The relationship between a fixed-point combinator and recursive functions is going to be illustrated by means of an example. We start by defining the plus function:

$$
\begin{aligned}
& x+y=y \text { if } x=0 ; \text { otherwise, } \\
& x+y=(x-1)+(y+1) .
\end{aligned}
$$

Using the reduction rules described above we arrive at

$$
\text { plus } \rightarrow \lambda x y \text {.if (iszero } x) y(\text { plus (pred } x)(\text { succ } y) \text { ) }
$$

A suitable term for plus is $Y M$, where $Y$ is the fixed-point combinator described earlier and $M$ is

$$
\lambda f \text {.xy.if (iszero } x) y(f(\text { pred } x)(\text { succ } y))
$$

In a similar way we can define a term times:

$$
Y \lambda f . x y \text {. if (iszero } x) 0 \text { (plus } y(f(\text { pred } x) y))
$$

By using plus and times we can define a well-known function: the factorial function.

$$
\text { factorial }=Y \lambda f . x \text {. if }(\text { iszero } x)(\text { succ } 0)(\text { times } x(f(\text { pred } x)))
$$

Restrictions on the use of constants are possible by introducing types into the lambda calculus. The notion of type is the same that we find in programming languages. For instance, an identity function from integers to integers is written as

$$
\lambda x: \text { integer. } x
$$


It is interesting to note that a function like identity might be defined not only for integers but also for reals or any other type. This means that identity should be defined as polymorphic function. In fact it is possible to write polymorphic functions in functional programming languages. The extended syntax for covering type allows a variable to be bound to a general type rather than a specific type like integer.

The lambda calculus is known to be computationally equivalent in power to many other plausible models for computation (including Turing machines); that is, any calculation that can be accomplished in any of these other models can be expressed in the lambda calculus, and vice versa.

\section{The library design}

\subsection{Formulas}

Formulas are expressions that can be manipulated in Algebra. They can be added and multiplied. They can be squared. Trigonometric transformations may be applied to them. They can be differentiated and integrated. There is a large number of operations that can be applied to formulas. These algebraic expressions are made up of numbers and variables, which can be combined in different ways.

Formulas can be grouped, according to their structure, in several classes, including

- Integers, correspond to the integer numbers, as defined in axiomatic theories like Peano's axioms, Church's numbers, or in one of the many brands of the Set Theory. A few examples of this class are 416, 7453 and -3291 .

- Constants, represent known mathematical entities. They are expressed by a symbol or name, like $\pi, e$ (neperian number) and i (imaginary unit). In general, a constant formula stands for a number without an exact representation in a given numeric system.

- Variables, correspond closely to mathematical variables. Logicians call them literals more often than not. Examples of literals: $x, y, a, b, \alpha, \beta$.

- Applications, which are formulas built from simpler formulas by applying a functional operator to arguments, which are formulas too. Exempli gratia: $\sin x, \operatorname{coth} x, \ln x, \log _{10} x, x+2,-a, \pi^{2}$ and $5 \times \sin [3 \pi(\alpha+2)]$. Applications may have different forms, depending on the operator. The basic arithmetic applications are

- Sum, denotes the sum of two formulas. Examples are $a+b$ and $13+y \times 4^{x}$.

- Product, denotes the product of two formulas. For example $4 \times x$ and $(a+b) \times(a+c)$.

- Power, denotes the power of two formulas, as in $x^{2}$ and $(a+b)^{\frac{1}{2}}$.

Other common applications are

- Logarithm of a formula in a given base. Examples are $\log _{10} y, \log _{e}(x+y)$, and $\log _{b} b^{c}$.

- Trigonometric formulas, like sines, cosines and tangent, of a formula. Examples: $\sin x, \cos a^{2}, \tan \left(x^{2}+y^{2}\right)$. There are many other possible forms of applications (hyperbolic formulas, derivatives, integrals, vectors, matrices, series, ...)

- Indeterminates, which are formulas whose value cannot be determined, like those obtained by dividing by zero. Examples are $\frac{3}{0}, 0^{0}$ and $\ln _{0} x$.

\subsection{Trivial representation of formulas}

The representation of formulas in the implementation language is tricky. One could simply use an algebraic data type to obtain the disjoint union of the relevant formula classes. Listing 2 is a first attempt in defining the type of (a simplified set of) formulas using this approach. It contemplates only the most basic classes of formulas: integers, named constants, variables, sums, products, powers and indeterminates.

Formulas that are applications have a common representation: all of them have an operator and a list of arguments. The type of the operator, $F n$, is just an enumeration of all possible operators: sum, product and power in our case. The arguments are formulas. For each possible class of formula there is a value constructor. 
Table 1 exemplifies mathematical formulas and their corresponding representation in the data type declared in listing 2.

\begin{tabular}{|c|c|}
\hline $\begin{aligned} \text { data Fn }=\text { Sum } & \text { Pro | Pow } \\
\text { data Formula } & =\text { Int Integer } \\
& \mid \text { Cte String } \\
& \text { | Var String } \\
& \text { | App Fn [Formula] } \\
& \text { Ind Formula }\end{aligned}$ & $\begin{array}{l}\text {-- sum, product or power } \\
\text {-- an integer } \\
\text {-- a known constant } \\
\text {-- a literal } \\
\text {-- an operator application } \\
\text {-- an indeterminate formula }\end{array}$ \\
\hline
\end{tabular}

Listing 2: A first attempt in building a type representation for formulas

In the representation, operators (that is, the value constructors) take a prefix form, where the constructor precedes the arguments. This prefix form is somewhat harder to read, but it eases the handling of expressions. Note that the mapping between the integer representation and the integer field is constrained only by the amount of memory available in the computer, as the |Integer| type represents arbitrary precision integers: its values can be as large as there is room for storing them in memory. There is no fixed number of bits for representing them as happens in conventional languages like C, Pascal and Java.

A naive addition operation is defined in listing 3. It is based on the following rules of Mathematics:

$$
\begin{aligned}
& m+n=\text { the sum of } m \text { and } n m, n \in Z, \\
& x+0=x, \\
& 0+x=x, \\
& (x+y)+z=x+y+z, \\
& x+(y+z)=x+y+z .
\end{aligned}
$$

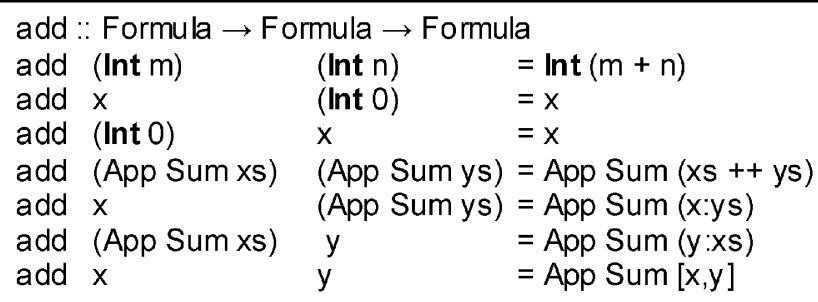

Listing 3: Addition of two formulas

Some identifiers we would like to use (such as $+, *, \wedge$ and $\sin$ ) are already bound in the Haskell standard libraries. So we will want to overload them. We also want to have some binary function names to have infix status. By doing so, we make sure that the notation used to express operations over formulas resembles the mathematical notation as far as possible, and the mathematical notation should be preferred in computational algebra systems.

A few examples of evaluation of formulas applying arithmetic operations follow.

$$
\begin{aligned}
& 2+\frac{3}{5}+\frac{8 x^{3}}{x}-x^{2} \Rightarrow \frac{13}{5}+7 x^{2}, \\
& a(a+b) \Rightarrow a^{2}+a b, \\
& \ln x^{5}-\ln x \Rightarrow 4 \ln x, \\
& \sin ^{2}(x+y)+\cos ^{2}(x+y) \Rightarrow 1 .
\end{aligned}
$$


Table 1

Representing mathematical formulas

\begin{tabular}{ll}
\hline Math & Haskell \\
\hline 831 & Int 831 \\
$\pi$ & Cte pi \\
$X$ & Var $x$ \\
$4+x$ & Sum [Int 4, Var $x]$ \\
$a \times x$ & App Pro [Var $a, \operatorname{Var} x]$ \\
$x^{2 \times a}$ & App Pow [Var $x$, App Pro [Int 2, Var $a]]$ \\
$x-y \times z$ & App Sum [Var $x$, App Pro [Int $(-1), \operatorname{Var} y, \operatorname{Var} z]]$ \\
$\pi / 2$ & App Pro [Cte pi, App Pow [Int 2, Int $(-1)]]$ \\
$m / 0$ & Ind (App Pro [Var $m$, App Pow [Int 0, Int $(-1)]])$ \\
\hline
\end{tabular}

The examples show that a computer algebra system is supposed to simplify any formula that cannot be reduced to a number. In general, an expression can be simplified in different ways, producing textually different but mathematically equal results. This issue is investigated in the next section. For the time being, we will take a look at Listing 4 which presents an example of using operations over formulas in a program. The example shows the symbolic solution of a quadratic equation. Note that symbolic variables are used exactly like numerical ones, making computation over formulas both easy and natural.

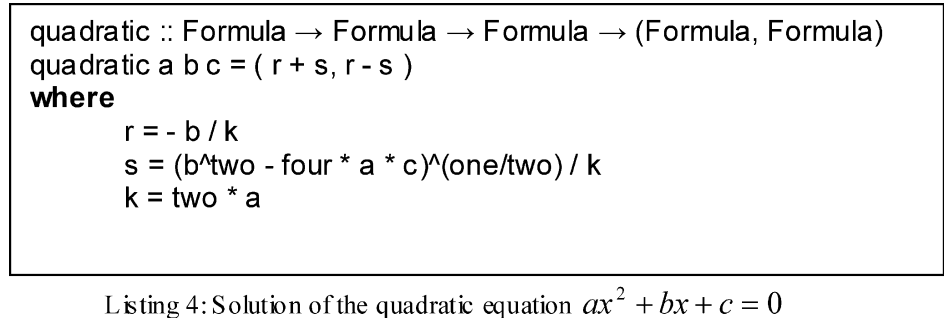

\subsection{Dealing with context}

An algorithm may need to evaluate an expression in many ways, depending on the desired form of the result. For example, the expression $b^{2} \cdot \frac{a}{b}+a a+b b+a b$ can be simplified to yield $a^{2}+2 a b+b^{2}$ or $(a+b)^{2}$ depending on whether we need to expand or to factor it. So the employed algorithms may produce different results. Therefore, when using an algorithm, one must state which kind of simplification is to be carried out. This is done by means of controllers, i.e., identifiers associated to values that control evaluation of formulas. The system may check these controllers at function applications, and build its result according to the values of the controllers. The derived set of controllers forms the context in which expressions are evaluated.

\subsection{Representing contexts}

The contexts are states represented as a record in the implementation language. Each controller is a field in the record. The value of a controller is the value of the corresponding field. Listing 5 shows an example of context. Appendix A briefly describes the meaning of some controllers. This initial context is bound to a global variable, since it may be used whenever one needs to restart a chain of state changes.

In the beginning of this section, it was said that context is a state representation. This means that the programmer may change one or more fields of the initial context. If we were dealing with a procedural language or even with a functional language with mutable data structures, we would change the global context directly. Haskell, like other pure functional languages, does not allow this, in order to guarantee referential transparency. However, one may pass the context as argument to a function and change it in the context of a new call. 


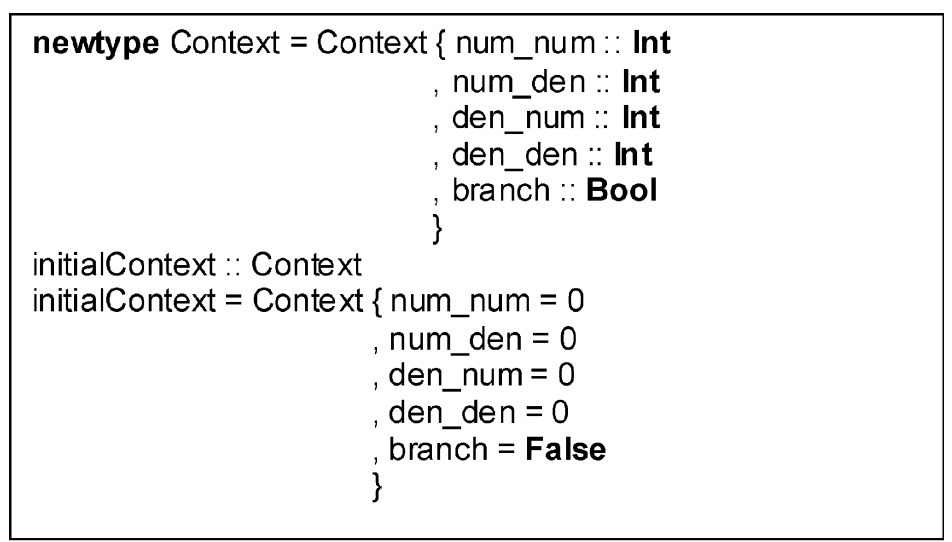

Listing 5: One may represent contexts as records

\section{Haskell limitations}

\subsection{Data type extensions}

The first limitation of Haskell is related to type extensibility. By that we mean the possibility of extending a data type with new value constructors in a new data type declaration. We highly desire a modular design, so a program that makes use of this system should mention only the relevant modules, i.e., a basic algebra and a linear algebra module. But the linear algebra module extends the $F n$ data type with new value constructors for the new formula formats it introduces, let us say Vec for vectors, and Mat for matrices. This cannot be accomplished in the current Haskell definition. And these new constructors have to be introduced in the module that defines the $F n$ data type.

If the user wants to build a new module to deal with a new kind of formula, let us say tensors, and needs to introduce a new value constructor, it has to be done by modifying the module defining $F n$ and we will have to distribute the source code of our library so that the user can recompile the modified module.

There are some research works that propose type extensibility for the Haskell type system. They include extensible union types [19], extensible records [20] and polymorphic subtyping [21].

\subsection{Function definition extension}

Another aspect of extendibility is the possibility of adding new equations for an already defined function. This is desired because, in our modular approach to the system design, we augment the definition of some functions as we deal with some new specific kinds of formulas.

For example, when implementing the algorithms related to trigonometric expressions (those involving sine and cosine) we need to add the rules for multiplication so that expressions like $2 \sin (x) \cos (x)$ can be transformed into $\sin (2 x)$. As we already have basic multiplication defined, we want to extend it to deal with such additional algorithms.

Haskell requires all the equations defining a function to be grouped (that is, in the same module, without any code intervening between them) and this greatly damages our modular design. At the end we had to have something like a big, really big, module with most of the definitions, or else, a collection of modules with very tight coupling, each one exporting many definitions that should be hidden in principle, and the general functions like the one implementing multiplication, dealing with all possibilities on a single module.

Again, if the user wants to extend the library with a new algorithm for a new kind of formula, the distributed modules will have to be edited and recompiled. Certainly this is completely against the desired modular design we would like to achieve. 


\subsection{Passing an environment around}

The problem of passing an environment around while a computation takes place certainly is not new to Haskell and to modern functional programming. But in our case it has some peculiarities.

As discussed before, the way formulas are manipulated depends on a context (environment) containing the controllers. With different environments, the same simplification may produce different results. So the environment has to be passed to the functions that implement the simplification algorithms in some way. At first it would be enough to add an extra argument corresponding to the environment of the relevant functions.

Passing the context as an extra argument is an acceptable solution, but it has a drawback: the arity of each function will be incremented by one. For instance, the binary functions will become ternary. The deleterious consequence of this increase in arity is that one cannot overload operators belonging to Haskell's primitive environment. For example, there will be no way of writing $x+y$ as the + function now needs a third argument. To solve this we tried the following:

1. Encapsulating the environment into the formulas themselves creating a new data type definition. This works well until we need to retrieve a controller in a function with more than one value as an argument: which one will be used as the environment supplier? There is no easy way to decide, as all of them carry an environment (the one that was visible when the value was constructed).

2. Making the function arguments not formulas, but a more elaborate construct: functions having the environment as input and the resulting formula as output. Then, the function would be called with some functional arguments which should be applied to an environment to find out the values on which to operate, and its result would be another function also to be applied to an environment to produce the desired result value. The definition would be something like the example from listing 6 . The problem with this solution is the loss of sharing among intermediate results [22]. To avoid this problem, one has to make the functional values memorize their results, which generally imposes drastic performance penalties on the system. This is the case unless there is a good library for function memorization that could be implemented independently to the specific Haskell implementation being used.

3. Implicitly passing the environment as an additional parameter to the functions, simulating dynamic scope from other languages [23]. In this way the additional parameter does not have to be written explicitly in the function calls, allowing one to use infix notation as desired. Some Haskell implementations offer this functionality as an extension.

Although Haskell supports mutable data structures without spoiling referential transparency as part of the monadic IO system, its use (as with any monad) imposes a sequential order of evaluation that is not desirable in a Computer Algebra system. Mathematics does not have any notion of order of evaluation. Putting one into our system would make it too artificial. Besides that, the use of monadic structures would make it too hard, or even impossible, to have a notation compatible with the common mathematical notation, that includes binary operators such as + and - .

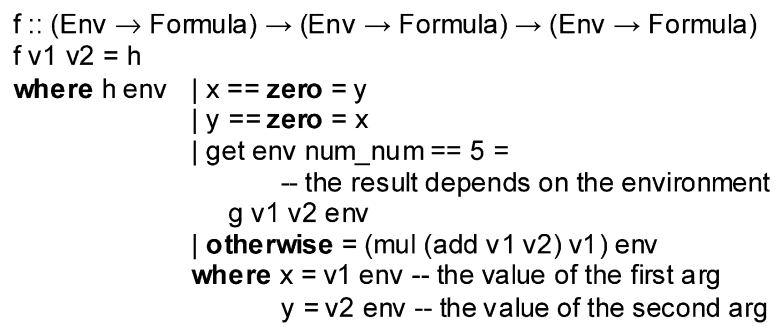

Listing 6: passing environments around 


\section{Conclusions}

Haskell, as a modern functional language, and with its referential transparency, is one of the best languages for implementing systems with high Mathematical backgrounds. Such a system has been developed by the authors and its development has demonstrated the viability of Haskell in real-world applications development. But some drawbacks in the design of Haskell have been met. It is therefore that these be removed in future revisions of the language.

The main problems we encountered are related to

- type extensibility

- function definition extensibility

- passing the environment around

To overcome these problems our design was not as sophisticated as we would like it to be and the modular principle was seriously damaged.

Currently there are some proposals of extensions to Haskell that would greatly reduce the difficulties we encountered, allowing for a better modular design of our system. We really look forward to seeing them in future versions of the language.

\section{Appendix A. The meaning of some controllers}

The algebraic controllers described in Section 4.4 enable the library user to apply fine control over the transformations used to simplify an expression.

The kinds of subformulas that are distributed or factored from a formula can be precisely controlled by setting an appropriate value to the appropriate controller. Positive integer values cause distribution whereas negative values cause factoring. The exact type of formula which is distributed or factored can be determined from Table 2.

If the value of the controllers num_num, den_num, num_den or den_den is an integer multiple of one of the primes listed in Table 2. Then formulas of the type associated with that prime are distributed (or factored if the controller is negative) in accordance with the transformations associated with that control variable.

- num_num - controls the distribution (factoring) of factors in the numerator of a formula over (from) the terms of a sum in the numerator using the transformation:

$$
a \times(b+c)=a \times b+a \times c
$$

Table 3 has an example of the use of num_num to control the distribution of factors over a sum. Note that differences are internally represented as sums involving negative coefficients.

- den_den - controls the distribution (factoring) of factors in the denominator of a formula over (from) the terms of a sum in the denominator using the transformation:

$$
\frac{1}{a \times(b+c)} \equiv \frac{1}{a \times b+a \times c}
$$

Table 2

Meaning of controllers

\begin{tabular}{lll}
\hline Prime & Type & Examples \\
\hline 2 & Numerical formulas & $4,-1 / 3,5 / 7$ \\
3 & Other nonsums & $x, \sin (x), z^{3}$ \\
5 & Sums & $r+s, x^{2}-x, \ln x+z$ \\
\hline
\end{tabular}


Table 3

Transforming $3 x \times(1+x)(1-x)$ with different values for num_num

\begin{tabular}{ll}
\hline num_num & Result \\
\hline 0 & $3 x \times(1+x)(1-x)$ \\
2 & $x \times(1+x)(3-3 x)$ \\
3 & $3(1+x)\left(x-x^{2}\right)$ \\
5 & $3 x \times(1+x-x \times(1+x))$ \\
6 & $(1+x)\left(3 x-3 x^{2}\right)$ \\
10 & $x \times(3+3 x+x \times(-3-3 x))$ \\
15 & $3\left(x-x^{3}\right)$ \\
30 & $3 x-3 x^{3}$ \\
\hline
\end{tabular}

For example, if den_den is 15 , the formula

$$
\frac{y}{x} \times \frac{1}{1+x} \times \frac{1}{1-x}
$$

is transformed into

$$
\frac{y}{x-x^{3}}
$$

- den_num - controls the distribution (factoring) of factors in the denominator of a formula over (from) the terms of a sum in the numerator using the transformation:

$$
\frac{b+c}{a} \equiv \frac{b}{a}+\frac{c}{a}
$$

For example, if den_num is 6 , the formula

$$
\frac{x+3}{\frac{3}{x}}
$$

is transformed into

$$
\frac{1}{3}+\frac{1}{x}
$$

- num_den - controls the distribution (factoring) of factors in the numerator of a formula over (from) the terms of a sum in the denominator using the transformation:

$$
\frac{a}{b+c} \equiv \frac{1}{\frac{b}{a}+\frac{c}{a}}
$$

This transformation yields a kind of continuation-fraction expansion. For example, if num_den is 5, the formula

$$
\frac{3+x}{1+x}
$$

is transformed into

$$
\frac{1}{\frac{x}{3+x}+\frac{1}{3+x}}
$$

If num_den is 30 , the result is

$$
\frac{1}{\frac{1}{1+\frac{3}{x}}+\frac{1}{3+x}}
$$




\section{References}

[1] F. Winkler, Polynomial Algorithms in Computer Algebra, Springer-Verlag, 1996.

[2] B. Buchberger, G.E. Collins, R. Loos (Eds.), Computer Algebra, Symbolic and Algebraic Computation, second ed., Springer, 1983.

[3] A.N. Whitehead, B. Russell, Principia Mathematica, vol. 1, Cambridge University Press, 1925, chapter Appendix 3.

[4] A. Diller, Compiling Functional Languages, John Willey \& Sons Ltd, 1988.

[5] B. Russel, Logic and Knowledge: Essays, George Allen \& Unwin, 1956.

[6] C.E. Delaunay, Théorie du Mouvement de la Lune, 2 Vols., Mem. Acad. Sci., 28 and 29, Gauthier-Villars, Paris, 1867.

[7] A. Deprit, J. Henrard, A. Rom, Ephemeris: Delaunay theory revisited, Science 168 (1970) 1569.

[8] N.J. Nilsson, Principles of Artificial Intelligence, Morgan Kaufman, 1980, pp. 35-47.

[9] C.M. Chang, Mathematical Analysis in Engineering, Cambridge University Press, 1994.

[10] A. Hearn, Reduce: A user-oriented interactive system for algebraic simplification, in: M. Klerer, J. Reinfelds (Eds.), Interactive Systems for Experimental Applied Mathematics, Academic Press, 1968, pp. 79-80.

[11] F. Brackx, D. Constales, Computer Algebra with LISP and REDUCE, Kluwer Academic Publishers, 1992.

[12] G. Andersson, Applied Mathematics with Maple, Chartwell-Bratt, 1997.

[13] D.C. Arney, Exploring Calculus with DERIVE, Addison-Wesley Publishing Company, 1992.

[14] G.L. Bradley, K.J. Smith, Calculus, Prentice Hall, 1995.

[15] A.D. Andrew, G.L. Cain, S. Crum, T.D. Morley, Calculus Projects Using Mathematica, McGraw-Hill, 1996.

[16] M. Schönfinkel, Uber die Bausteine der mathematischen Logik, Mathematische Annalen 92, 305-316.

[17] H.B. Curry, R. Feynes, Combinatory Logic, vol. 1, North-Holland, Amsterdam, 1958.

[18] A. Church, The calculi of lambda conversion, Annals of Math. Studies, vol. 6, Princeton University Press, Princeton, NJ, 1941.

[19] S. Liang, P. Hudak, M. Jones, Monad transformers and modular interpreters, in: Conference Record of POPL'95: 22nd ACM SIGPLAN- SIGACT Symposium on Principles of Programming Languages, San Francisco, CA, January 1995.

[20] M.P. Jones, S.P. Jones, Lightweight extensible records for haskell, in: Proceedings of the 1999 Haskell Workshop, October 1999.

[21] J. Nordlander, Polymorphic subtyping in o'haskell, in: Proceedings of the APPSEm Workshop on Subtyping and Dependent Types in Programming, Ponte de Lima, Portugal, 2000.

[22] S.P. Jones, S. Marlow, C. Elliott, Stretching the storage manager: weak pointers and stable names in haskell, IFL, 1999.

[23] J. Lewis, M. Shields, E. Meijer, J. Launchbury, Implicit parameters: Dynamic scoping with static types, in: 27th Annual ACM SIGPLAN-SIGACT Symposium on Principles of Programming Languages (POPL'00), 2000. 